\title{
Viscosity and mutual diffusion in strongly asymmetric binary ionic mixtures
}

\author{
Sorin Bastea* \\ Lawrence Livermore National Laboratory, P.O. BOX 808, Livermore, CA 94550
}

\begin{abstract}
We present molecular dynamics simulation results for the viscosity and mutual diffusion constant of a strongly asymmetric binary ionic mixture (BIM). We compare the results with available theoretical models previously tested for much smaller asymmetries. For the case of viscosity we propose a new predictive framework based on the linear mixing rule, while for mutual diffusion we discuss some consistency problems of widely used Boltzmann equation based models.

PACS numbers: 52.25.Fi, 52.27.Cm, 52.27.Gr
\end{abstract}

The important advancements that occurred in the last decade in the experimental techniques involving high-power lasers have generated a renewed interest in the properties of dense plasmas in general and their transport properties in particular [1, 2, 3]. The experimental capabilities currently available and the ones that are expected to become available in the near future [4] promise to further advance the field of inertial confinement fusion (ICF) as well as shed new light on long-standing astrophysics problems. Many times such experiments either probe, or their outcome is strongly dependent on, the behavior of plasma mixtures with various degrees of charge and mass asymmetries of the components. Such mixtures occur for example in ICF due to the instability (e.g. Richtmyer-Meshkov or RayleighTaylor) driven mixing of the heavy elements that make up the enclosing shell and the much lighter fuel. In this case the stability of the initial interfaces, nature of the ensuing flows and degree of fuel contamination are crucially linked to such mixture properties as shear viscosity and mutual diffusion [ [, 6, 7]. In the present paper we calculate these properties using molecular dynamics simulations for a simple but relevant plasma model: the binary ionic mixture (BIM) [8, 9], which is a generalization of the one-component plasma (OCP) 10]. We study a rather extreme, ICFinspired system [7], $D^{+}-A u^{39+}$, which displays roughly two orders of magnitude charge and mass asymmetry, but the results should apply to other plasma mixtures as well, e.g. of astrophysics significance [11], where large charge and mass asymmetries are present. We compare the MD results with available theoretical models previously tested for much smaller asymmetries. For the case of viscosity we propose a new predictive framework based on the linear mixing rule, while for mutual diffusion we discuss some consistency problems of Boltzmann equation based models.

The BIM model consists of a mixture of $N_{1}$ point ions of charge $q_{1}=Z_{1} e$ and mass $M_{1}$ and $N_{2}$ point ions of charge $q_{2}=Z_{2} e$ and mass $M_{2}$ embedded in a uniform, rigid, neutralizing electronic background. We denote the number concentrations by $x_{\alpha}=N_{\alpha} / N, N=N_{1}+N_{2}, \alpha=1,2$ and number densities by $\rho_{\alpha}=N_{\alpha} / V, \rho=\rho_{1}+\rho_{2}$, where $V=L^{3}$ is the volume. $\langle Z\rangle=x_{1} Z_{1}+x_{2} Z_{2}$ is the average charge and $\rho \prime=\rho_{1} Z_{1}+\rho_{2} Z_{2}$ the electronic number density. As usual the mean inter-electronic and inter-ionic distances are defined by $a \prime=(3 / 4 \pi \rho)^{\frac{1}{3}}$ and $a=(3 / 4 \pi \rho)^{\frac{1}{3}}=a \prime\langle Z\rangle^{\frac{1}{3}}$, while the electronic and ionic coupling parameters are:

$$
\begin{aligned}
\Gamma^{\prime} & =\frac{e^{2}}{a / k_{B} T}=\Gamma\langle Z\rangle^{\frac{1}{3}} \\
\Gamma_{i} & =\Gamma^{\prime}\left\langle Z^{\frac{5}{3}}\right\rangle
\end{aligned}
$$

with

$$
\Gamma=\frac{e^{2}}{a k_{B} T}
$$

The thermodynamics of the system is fully determined by one coupling constant, e.g. $\Gamma$, and concentration $x_{1}$. As it is the case for the OCP, the validity range of the BIM model is such that the Fermi temperature of the electrons is $T_{F} \gg T$ and the densities are high enough so $r_{s} \ll 1, r_{s}=a l / a_{0}, a_{0}$ - Bohr radius, corresponding to a completely degenerate and rigid electronic background.

The thermodynamics of the BIM has been thoroughly studied and is known to be very well described by the linear mixing rule 8, 12, 13, 14]. For moderate charge asymmetries an OCP-based "one-fluid theory" is also a reasonable approximation [8], with an effective charge $Z_{\text {eff }}^{2}=\left\langle Z^{\frac{5}{3}}\right\rangle\langle Z\rangle^{\frac{1}{3}}$ suggested by the ion-sphere model [15]. The relative success of this "one-fluid" representation has lead Clerouin et al. to propose that the shear viscosity can also be predicted in terms of the equivalent OCP, as already tested for thermal transport [16]. (Although not explicitly

*Electronic address: bastea2@llnl.gov 
stated in [2], further assumptions need to be made for such a prediction - see below). This "one-fluid" approach was shown to be suitable for calculating the BIM viscosity at charge and mass asymmetries of order $\approx 10$. Before testing this idea on much larger asymmetries, $\approx 100$, we note that, surprisingly, the viscosity of the OCP itself does not appear to be very accurately known. For intermediate and strong couplings, $1 \leq \Gamma \leq 100$, Bernu and Vieillefosse 17] have proposed an interpolation formula based on three MD simulation results obtained with systems of 128 250 particles, while at even stronger couplings Ichimaru and Tanaka have introduced a generalized viscoelastic theory [18]. In [2] the authors propose a different relation based on the kinetic theory of Wallenborn and Baus [19], that extends to the weak coupling regime. However, the disagreement between these two approaches (Refs. 17] and [2]) is significant in the regime that they both cover, $\Gamma \geq 1$, particularly at intermediate $\Gamma$ 's. Unfortunately it is difficult to ascertain the reliability of these predictions given both the limited simulation results available and the small system size used, which limits the accuracy of the results.

To settle this question we performed extensive microcanonical MD simulations of the OCP with much larger system sizes - 1372 particles, and a wide range of coupling constants, $0.05 \leq \Gamma \leq 100$. The Coulomb interactions were handled using the Ewald summation technique with conducting boundary conditions. The calculation of the shear viscosity $\eta$ was done using the Green-Kubo relation:

$$
\eta=\frac{1}{V k_{B} T} \int_{0}^{\infty}\left\langle\hat{\sigma}_{x y}(t) \hat{\sigma}_{x y}(0)\right\rangle d t
$$

As shown by Bernu and Vieillefosse [17], and more recently in the context of Yukawa plasmas by Salin and Caillol [20], the evaluation of the pressure tensor $\hat{\sigma}$ requires an Ewald-type summation for its interaction part, $\hat{\sigma}^{I}$ :

$$
\begin{aligned}
& \hat{\sigma}=\hat{\sigma}^{K}+\hat{\sigma}^{I} \\
& \hat{\sigma}_{a b}^{K}=\sum_{i} M_{i} v_{i, a} v_{i, b} \\
& \hat{\sigma}^{I}=\hat{\sigma}^{(r)}+\hat{\sigma}^{(k)} \\
& \hat{\sigma}_{a b}^{(r)}=\frac{1}{2} \sum_{i \neq j} q_{i} q_{j} \frac{r_{i j, a} r_{i j, b}}{r_{i j}}\left[\frac{2 \alpha e^{-\alpha^{2} r_{i j}^{2}}}{\sqrt{ } \pi r_{i j}}+\frac{\operatorname{erfc}\left(\alpha r_{i j}\right)}{r_{i j}^{2}}\right] \\
& \hat{\sigma}_{a b}^{(k)}=\frac{2 \pi}{L^{3}} \sum_{|\mathbf{k}| \neq 0} \frac{e^{-\frac{k^{2}}{4 \alpha^{2}}}}{k^{2}}\left[\delta_{a b}-2\left(1+\frac{k^{2}}{4 \alpha^{2}}\right) \frac{k_{a} k_{b}}{k^{2}}\right]|\tilde{\rho}(\mathbf{k})|^{2} \\
& \tilde{\rho}(\mathbf{k})=\sum_{i} q_{i} e^{-i \mathbf{k} \cdot \mathbf{r}_{\mathbf{i}}}
\end{aligned}
$$

where $a$ and $b$ denote the Cartesian coordinates. The Ewald parameter $\alpha$ 21] was chosen such that the real space sums, e.g. $\hat{\sigma}^{(r)}$, can be calculated with the usual minimum-image convention, as shown above. The duration of the runs was $10^{3}-10^{4} \omega_{p}^{-1}$, where $\omega_{p}^{2}=4 \pi \rho e^{2} / M$ is the plasma frequency $(Z=1)$. The natural unit for the viscosity of the OCP is $\eta_{0}=\rho M a^{2} \omega_{p}$.

Our simulation results are shown in Fig. 1 together with the interpolation formula of Bernu and Vieillefosse (for $\Gamma \geq 1$ ) and the relation proposed in [2] based on OCP kinetic theory. The errors, estimated using a standard block analysis [22], are between about $8 \%$ at intermediate and large $\Gamma$ and $25 \%$ at the lowest $\Gamma$. The present viscosity results largely agree with those of [17], but suggest that in the intermediate coupling range the OCP viscosity is significantly higher than previously predicted, in agreement with [23]. The well known viscosity minimum appears to be around $\Gamma \simeq 21$, with $\eta / \eta_{0} \simeq 0.084$. We are not aware of other simulations for weakly coupled plasmas, $\Gamma \leq 1$, but our viscosity results in this regime are in qualitative agreement with the kinetic theory of Wallenborn and Baus, although somewhat lower. Since for these conditions the screened, Debye-Hückel potential $e^{2} \exp \left(-r / \lambda_{D}\right) / r, \lambda_{D}=\left[k_{B} T / 4 \pi e^{2} \rho\right]^{\frac{1}{2}}$, should be an appropriate representation of the effective inter-ionic interaction and $a / \lambda_{D} \propto \Gamma^{\frac{1}{2}}$, i.e. the interaction can be fairly short-ranged even for $\Gamma$ significantly smaller than 1 , a good viscosity estimate should be provided by the

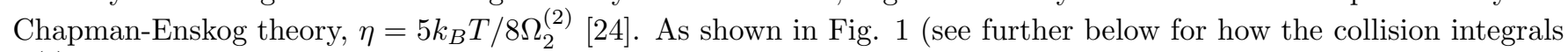
$\Omega_{2}^{(2)}$ are calculated) this is indeed the case for $0.05 \leq \Gamma \leq 1$. We choose to fit all the data points with the relation:

$$
\frac{\eta}{\eta_{0}}=A \Gamma^{-2}+B \Gamma^{-s}+C \Gamma
$$

which captures rather well the behavior of the OCP viscosity in the wide range of couplings simulated. The best parameters are: $A=0.482, B=0.629, C=1.88 \times 10^{-3}$, and $s=0.878$. 
We know tackle the question of the BIM viscosity, in particular when the charge and mass asymmetries are very large. It should be noted that the system that we focus on, $D^{+}-A u^{39+}$, is not a simple BIM per se, as gold $(A u)$ is only partially ionized and the effect of the remaining electrons may be important under certain thermodynamic conditions. However, the BIM approach is still relevant provided the densities and temperatures are such that the distance of closest approach between ions is larger that the radius of the remaining ion cores.

To elucidate the effect on viscosity of mixing plasmas with such large differences in charge and mass as $D^{+}$and $A u^{39+}$ it is convenient to adopt the procedure of Ref. [2], where the coupling constant $\Gamma$ is kept fixed and the concentration of the two species is varied. We set $\Gamma=0.05$, which corresponds to $\Gamma_{i}=0.05$ for pure deuterium $\left(x_{A u}=0\right)$, i.e. a weakly coupled plasma, and to $\Gamma_{i} \simeq 76$ for pure gold $\left(x_{A u}=1\right)$, i.e. a strongly coupled plasma. As before, we perform microcanonical simulations with a system of 1372 particles, at a number of different concentrations $x_{A u}$. Due to the strong charge and mass asymmetries, exceedingly long run times are necessary for both equilibration and data accumulation to calculate the viscosity using Eq. 4 with an accuracy of $20-25 \%$. A good measure of the large "size" difference between the ions is provided for example by the pair correlation functions, which we show in Fig. 2 for $x_{A u}=0.5$.

As noted in 2] the viscosity drops steeply upon mixing highly charged, heavy ions in a weakly coupled plasma, see Fig. 3 This effect can be understood qualitatively in the framework of a "one-fluid" theory, which we describe below. The coupling constant of the equivalent OCP is $\Gamma_{\text {eff }}=\Gamma Z_{\text {eff }}^{2}$, where $Z_{\text {eff }}^{2}=\left\langle Z^{\frac{5}{3}}\right\rangle\langle Z\rangle^{\frac{1}{3}}$ follows from the ion-sphere model [15]. In this approximation the thermodynamics is fully determined by $\Gamma_{e f f}$, but the calculation of the viscosity requires some additional arguments. For example, a reasonable unit for the viscosity of this system may be assumed to be $\eta_{0}^{m}=\rho\langle M\rangle a^{2} \omega_{p m},\langle M\rangle=x_{1} M_{1}+x_{2} M_{2}$, where $\omega_{p m}^{2}=\omega_{p}^{2}\langle Z\rangle^{2} /\langle M\rangle$ is the "hydrodynamic" plasma mixture frequency. (The use of the so-called "kinetic" mixture frequency [25] leaves the results largely unchanged). The mixture viscosity in these units is then postulated to be given by the scaled effective-OCP viscosity:

$$
\frac{\eta(\Gamma, x)}{\eta_{0}^{m}(x)}=\frac{\eta_{O C P}\left(\Gamma_{e f f}\right)}{\eta_{0}}
$$

We show in Fig. 3 the results of such calculations for the system that we study, using both the OCP viscosity of Ref. 2], and our prediction Eq. 11] The qualitative dependence of the MD results on $x_{A u}$, arising from the opposite behaviors of $\eta_{O C P}\left(\Gamma_{e f f}\right)$ and $\eta_{0}^{m}(x)$, is reproduced correctly, but the quantitative disagreement is also very significant, particularly for small and moderate $A u$ concentrations. The use of the more accurate OCP viscosity Eq. 11] does not fully alleviate this problem. We conclude that, not surprisingly, the accuracy of the "one-fluid" model is diminished for extreme asymmetries.

It is clear that this limitation can only be overcome by taking into account, either explicitly or implicitly, the mixture asymmetry. A direct calculation along the lines of the kinetic theory of Wallenborn and Baus [19] has been used for example to determine the BIM mutual diffusion constant at small asymmetries [26]. However, the calculation of the viscosity is even more complex and given the limitations of the theory even for the OCP its success for BIM quantitative predictions is rather doubtful. We turn therefore to a more indirect method, which we outline below. First, we recall an interesting and much studied property of binary ionic mixtures, the linear mixing rule [8, 12, 13, 14]. Hansen et al. have pointed out that the excess internal energy of the BIM, $u=U_{e x} / N k_{B} T$, is very acurately represented as a linear combination of the excess energies of two one-component plasmas with the same electronic coupling constant $\Gamma^{\prime}$ as the BIM (and ionic charges $Z_{1} e$ and $Z_{2} e$ ), i.e. the mixing of the two components at the same temperature and electronic density is largely ideal:

$$
u\left(\Gamma^{\prime}, x_{1}\right) \simeq x_{1} u_{O C P}\left(\Gamma^{\prime} Z_{1}^{\frac{5}{3}}\right)+x_{2} u_{O C P}\left(\Gamma^{\prime} Z_{2}^{\frac{5}{3}}\right)
$$

We find that this rule is satisfied at very large asymmetries as well, with the largest relative deviations occurring at $\Gamma^{\prime} \ll 1$, i.e. small $A u$ concentrations, in agreement with [8]. Given this nearly ideal mixing behavior we assume that other system properties, e.g. viscosity, are bracketed by the component values as well. For an interpolation relation between the viscosities of the two OCP's at a given composition we now borrow some concepts from the linear transport theory of composite media. A common situation encountered in such systems is that of "impurities" with a generalized conductivity $\alpha_{1}$ and total volume fraction $\phi_{1}$ randomly dispersed in a matrix $\alpha_{2}$. Under these circumstances the effective medium theory [27] employs a mean-field like, self-consistent approximation to predict the medium conductivity on scales much larger than those of the inhomogeneities. For the case of viscosity this yields [28] for the effective medium viscosity $\eta_{m}$ :

$$
\sum_{i} \phi_{i} \frac{\eta_{i}-\eta_{m}}{\eta_{i}+\frac{3}{2} \eta_{m}}=0
$$

We now note that the BIM is obtained by combining one-component plasmas with volume fractions $\phi_{i}=Z_{i} x_{i} /\langle Z\rangle$ and use the above relation to predict the mixture viscosity, essentially assuming that the theory applies for atomically 
sized "impurity" domains. Using Eq. 11 for the individual OCP viscosities $\eta_{1}$ and $\eta_{2}$ we obtain the results shown in Fig. [3.

The degree of agreement with the simulation results is fairly remarkable. This may lead one to believe that the system is perhaps thermodynamically unstable and separating into two OCP phases [8]. However, we find no evidence for such a scenario and conclude that the decoupling signaled by the linear mixing rule along with the tremendous asymmetry between the ions lead to behavior mimicking that of a macroscopically mixed system.

We now turn to the case of ionic interdiffusion in the BIM. Mutual diffusion in plasma mixtures plays an important role in the prediction of stellar structure [29], as well as the stability of ICF targets [5]. For the case of a binary mixture the mutual diffusion coefficient $D_{12}$ can be calculated in terms of the fluctuations of the microscopic interdiffusion current [30]:

$$
\begin{aligned}
D_{12} & =x_{1} x_{2}\left[\frac{\partial^{2}(\beta G / N)}{\partial x_{1}^{2}}\right]_{P, T} D_{12}^{0} \\
D_{12}^{0} & =\frac{1}{3 N x_{1} x_{2}} \int_{0}^{\infty}\left\langle\mathbf{j}_{c}(t) \mathbf{j}_{c}(0)\right\rangle d t \\
\mathbf{j}_{c}(t) & =x_{2} \sum_{1}^{N_{1}} \mathbf{v}_{i}(t)-x_{1} \sum_{1}^{N_{2}} \mathbf{v}_{i}(t)
\end{aligned}
$$

where $G$ is the Gibbs free energy. The thermodynamic prefactor that multiplies the Green-Kubo component $D_{12}^{0}$ reduces to unity for dilute gas mixtures [30], but in low density, weakly coupled plasmas goes to $\left\langle Z^{2}\right\rangle /\langle Z\rangle^{2}$, which has been interpreted as an effect of the ambipolar electric field of the electrons [26]. It is worth noting that the above relation is a good estimate for $x_{1} x_{2}\left[\partial^{2}(\beta G / N) / \partial x_{1}^{2}\right]_{P, T}$ at weak as well as strong couplings. Since the linear mixing rule holds well for all couplings, the change in Helmholtz free energy upon mixing at constant electronic density (and temperature):

$$
\frac{\Delta F}{N}=f\left(\Gamma^{\prime}, x_{1}\right)-x_{1} f_{O C P}\left(\Gamma^{\prime} Z_{1}^{\frac{5}{3}}\right)-x_{2} f_{O C P}\left(\Gamma^{\prime} Z_{2}^{\frac{5}{3}}\right)
$$

is very well approximated by the ideal entropy of mixing (with negative sign):

$$
\frac{\Delta F}{N} \simeq k_{B} T\left[x_{1} \ln \frac{x_{1} Z_{1}}{\langle Z\rangle}+x_{2} \ln \frac{x_{2} Z_{2}}{\langle Z\rangle}\right]
$$

If we assume that the system pressure $p=p_{\text {electronic }}+p_{\text {ionic }}$ is entirely determined by the electronic density, i.e. $p_{\text {electronic }} \gg p_{\text {ionic }}$, which is consistent with the initial assumption of a rigid electronic background, $r_{s} \ll 1$, then $\Delta G=$ $\Delta F$ [8]. We can therefore immediately calculate the thermodynamic prefactor as $\left\langle Z^{2}\right\rangle /\langle Z\rangle^{2}$. The difference between BIM and ideal gas mixtures appears here to be an entropic effect induced by the charge neutralizing background, as mixing occurs at constant electronic density (i.e. constant electronic pressure) as opposed to constant molecular density (i.e. constant ideal gas pressure).

In the course of the molecular dynamics simulations with various $D^{+}-A u^{39+}$ mixtures we also calculated the microscopic interdiffusion current $\mathbf{j}_{\mathbf{c}}$, and therefore were able to determine the Green-Kubo integrand $D_{12}^{0}$. The results are shown in Fig. 4 relative to $D_{0}=a^{2} \omega_{p}$, along with the discussed thermodynamic prefactor estimate. We find that $D_{12}^{0}$ is almost concentration independent for $x_{A u} \geq 0.1$, but appears to decrease fairly steeply at lower concentrations. The prefactor $\left\langle Z^{2}\right\rangle /\langle Z\rangle^{2}$ has a simple behavior, with a sharp maximum for small amounts of highly charged ions [31].

For a BIM with small ionic asymmetry kinetic theory estimates of the $D_{12}^{0}$ were found to be in good agreement with simulations [26]. A simpler model, widely employed for astrophysics problems, was proposed by Paquette et al. [32]. Its main assumption is that the Boltzmann equation can be used to calculate the transport coefficients of plasma mixtures modeled as BIM by making use of the Chapman-Enskog solution method 24]. The authors further argue that screened potentials, $Z_{i} Z_{j} e^{2} \exp (-r / \lambda) / r$, are better suited for such estimates than the pure Coulomb interaction. In order to extend the validity of this approach to strong couplings they propose as appropriate screening distance $\lambda$ the larger of $\lambda_{D}$ and $a$, where $\lambda_{D}$ is the Debye screening length:

$$
\lambda_{D}=\left[\frac{k_{B} T}{4 \pi e^{2}\left(\rho^{\prime}+\sum_{i} \rho_{i} Z_{i}^{2}\right)}\right]^{\frac{1}{2}}
$$

Under these assumptions the Boltzmann equation mutual diffusion coefficient is given in the first Enskog approximation as:

$$
\left[D_{12}\right]_{1}=\frac{3 k_{B} T\left(M_{1}+M_{2}\right)}{16 \rho M_{1} M_{2} \Omega_{12}^{(11)}}
$$


where $\Omega_{12}^{(11)}$ are collision integrals [24] that have been tabulated with high accuracy in Ref. [32]. We perform such calculations for the $D^{+}-A u^{39+}$ mixture using the slightly better second Enskog approximation 32]. The outcome, see Fig. 4 reproduces rather well the MD simulation results for $D_{12}^{0}$ at $x_{A u} \geq 0.1$, but not the mutual diffusion coefficient $D_{12}$. This is an important point that merits further discussion. In fact, there is no reason to expect that Chapman-Enskog estimates based on the Boltzmann equation for the ions can reproduce the full $D_{12}$ for either pure Coulomb interactions (with some reasonable cut-off) or screened potentials. As shown in 26] for low density plasmas or more generally here, the prefactor value $\left\langle Z^{2}\right\rangle /\langle Z\rangle^{2}$ only arises when the electronic background is explicitly taken into account either through its ambipolar field in a dilute plasma kinetic description [26] or simply at the thermodynamic level in the context of the linear mixing rule. No such effect is included when the standard Boltzmann equation, which is consistent with ideal gas thermodynamics, is used to model the dynamics of the ions. Therefore it is reasonable to expect that such approaches can only provide estimates of the Green-Kubo part, i.e. $D_{12}^{0}$, of the mutual diffusion constant, as already evidenced by our simulation results and perhaps not fully appreciated before. We note however that the prefactor value quoted above may be a good approximation only for $r_{s} \rightarrow 0$. For real systems, e.g. those encountered in ICF or astrophysics problems, $r_{s}$ departs significantly from zero and the electronic pressure and polarization effects can lead to phase separation 8, 33], especially at high asymmetries. The thermodynamic quantity $\left(\partial^{2}(\beta G / N) / \partial x_{1}^{2}\right)_{P, T}$ is connected to the spinodal decomposition line of the plasma mixture 33], and therefore proper estimates require careful calculations. In particular, it was pointed out in [33] that it is not sufficient to consider a BIM with compressible but non-polarizable electronic background since a consistent treatment can only be achieved with an appropriate modeling of electronic screening.

The apparent failure at low $A u$ concentrations of the screened potentials method proposed by Paquette et al. may appear at first puzzling since as $x_{A u}$ decreases so does the effective coupling constant $\Gamma_{\text {eff } f}$, and therefore the accuracy of the theory should in principle only increase. We note however that this also requires $\lambda_{D}>a$, which for our system and chosen $\Gamma$ means $x_{A u}$ no bigger than $\approx 10^{-3}$. In fact, although not easily seen in Fig. 4 the theoretical values drop sharply for such compositions to values close to the MD result at the lowest simulated $A u$ concentration, $x_{A u}=0.03$. For this composition the screening distance $a$, although larger than $\lambda_{D}$, appears to be too small. This is perhaps not unexpected given that due to the significant separation between the highly-charged ions they are primarily screened by the small ions and the electronic background, which requires distances significantly larger than $a$ when the charge asymmetry is very large.

In summary, we have investigated using molecular dynamics simulations the viscosity and mutual diffusion coefficients of a plasma model known as the binary ionic mixture (BIM) when the asymmetry is very strong. We discuss in light of the MD results important shortcomings of widely used theoretical models at large asymmetries. For viscosity, an OCP-based "one-fluid" theory proves inadequate for quantitative predictions and we propose a new method based on the linear mixing rule. A commonly employed model of ionic interdiffusion based on the Boltzmann equation compares reasonable well with the simulation results, but we point out that it provides only part of the mutual diffusion coefficient. The missing thermodynamic piece may be particularly important for large asymmetries and low concentrations of highly-charged ions, situations often encountered in both ICF and astrophysics applications. Finally, calculations that take into account electronic polarization effects are currently under way and will be reported in a future publication.

I thank Harry Robey, Peter Amendt and Jose Milovich for informative discussions. This work was performed under the auspices of the U. S. Department of Energy by University of California Lawrence Livermore National Laboratory under Contract No. W-7405-Eng-48.

[1] Y. Rosenfeld, E. Nardi, Z. Zinamon, Phys. Rev. Lett. 75, 2490 (1995).

[2] J.G. Clérouin, M.H. Cherfi, G. Zérah, Europhys. Lett. 42, 37 (1998).

[3] G. Salin, J.-M. Caillol, Phys. Rev. Lett. 88, 065002 (2002).

[4] J.A. Paisner, J.D. Boyes, S.A. Kumpan, W.H. Lowdermilk, M.S. Sorem, Laser Focus World 30, 75 (1994).

[5] H.F. Robey, Y. Zhou, A.C. Buckingham, P. Keiter, B.A. Remington, R.P. Drake, Phys. Plasmas 10, 614 (2003).

[6] P. Amendt, J.D. Colvin, J.D. Ramshaw, H.F. Robey, O.L. Landen, Phys. Plasmas 10, 820 (2003).

[7] J.L. Milovich, P. Amendt, M. Marinak, H. Robey, Phys. Plasmas 11, 1552 (2004).

[8] J.P. Hansen, G.M. Torrie, P. Vieillefosse, Phys. Rev. A 16, 2153 (1977).

[9] J.P. Hansen, I.R. McDonald, P. Vieillefosse, Phys. Rev. A 20, 2590 (1979).

[10] J.P. Hansen, Phys. Rev. A 8, 3096 (1973).

[11] N.S. Schulz, C. Canizares, D. Huenemoerder, K. Tibbets, Astrophys. J. 595, 365 (2003).

[12] S. Ogata, H. Iyetomi, S. Ichimaru, H.M. Van Horn, Phys. Rev. E 48, 1344 (1993).

[13] H. DeWitt, W. Slattery, G. Chabrier, Physica B 228, 21 (1996).

[14] H.E. DeWitt, W.L. Slattery, G.S. Stringfellow, in Strongly coupled plasmas, edited by S. Ichimaru, p.635 (Elsevier Science, 
New York, 1990).

[15] E.E. Salpeter, Aust. J. Phys. 7, 353 (1954).

[16] C. Pierleoni, G. Ciccotti, J. Phys.-Condens. Mat. 2, 1315 (1990).

[17] B. Bernu, P. Vieillefosse, Phys. Rev. A 18, 2345 (1978).

[18] S. Ichimaru, S. Tanaka, Phys. Rev. Lett. 56, 2815 (1986).

[19] J. Wallenborn, M. Baus, Phys. Rev. A 18, 1737 (1978).

[20] G. Salin, J.-M. Caillol, Phys. Plasmas 10, 1220 (2003).

[21] See, e.g., M. Deserno, C. Holm, J. Chem. Phys. 109, 7678 (1998).

[22] M.P. Allen, D.J. Tildesly, Computer Simulation of Liquids, (Clarendon Press, Oxford, 1987).

[23] Z. Donkó, B. Nyíri, Phys. Plasmas 7, 45 (2000).

[24] S. Chapman, T.G. Cowling, The Mathematical Theory of Non-uniform Gases (Cambridge University Press, Cambridge, England, 1970).

[25] J.P. Hansen, F. Jolly, I.R. McDonald, Physica 132A, 472 (1985).

[26] D.B. Boercker, E.L. Pollock, Phys. Rev. A 36, 1779 (1987).

[27] R. Landauer, J. Appl. Phys. 23, 779 (1952).

[28] See, e.g., F. Lequeux, A. Ajdari, Phys. Rev. E 63, 030502 (2001).

[29] S. Vauclair, Space Sci. Rev. 84, 265 (1998).

[30] J.-P. Hansen, I.R. McDonald, Theory of Simple Liquids, $2^{\text {nd }}$ edition, (Academic Press, London, 1986).

[31] The qualitative behavior of the thermodynamic prefactor remains unchanged (but its values are even higher) if deviations from the linear mixing rule are taken into account and estimated using the relation proposed in Ref. [12] (the present charge assymmetry is however larger than the ones studied in [12]).

[32] C. Paquette, C. Pelletier, G. Fontaine, G. Michaud, Astrophys. J., Suppl. Ser. 61, 177 (1986).

[33] D. Léger, C. Deutsch, Phys. Rev. A 37, 4916 (1988). 


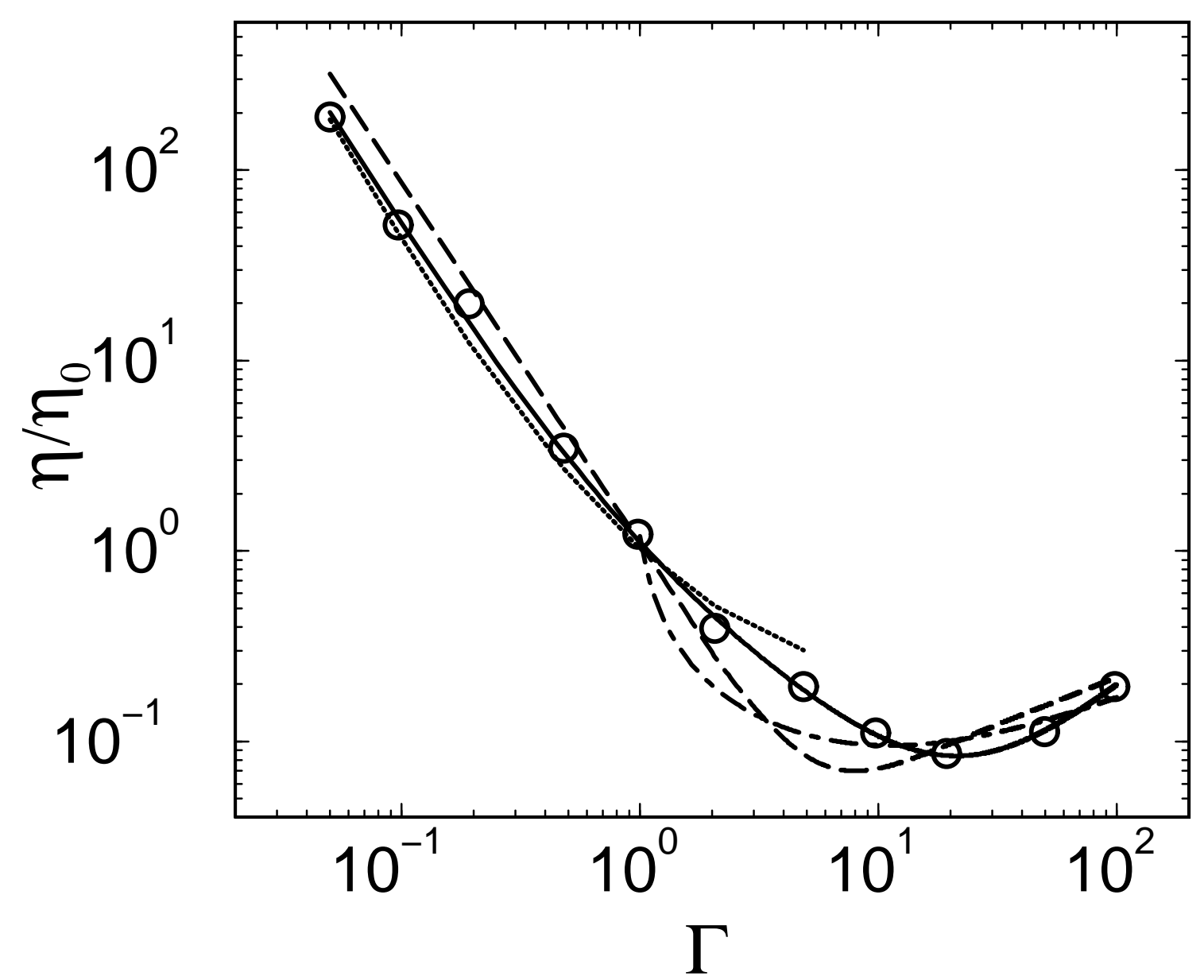

FIG. 1: OCP viscosity: present simulations (circles), fit of simulation results - Eq. 11] (solid line), predictive relation of Ref. [2] (dashed line), Bernu-Vieillefosse interpolation formula [17] (dot-dashed line), Chapman-Enskog estimate using screened potentials (dotted line). 


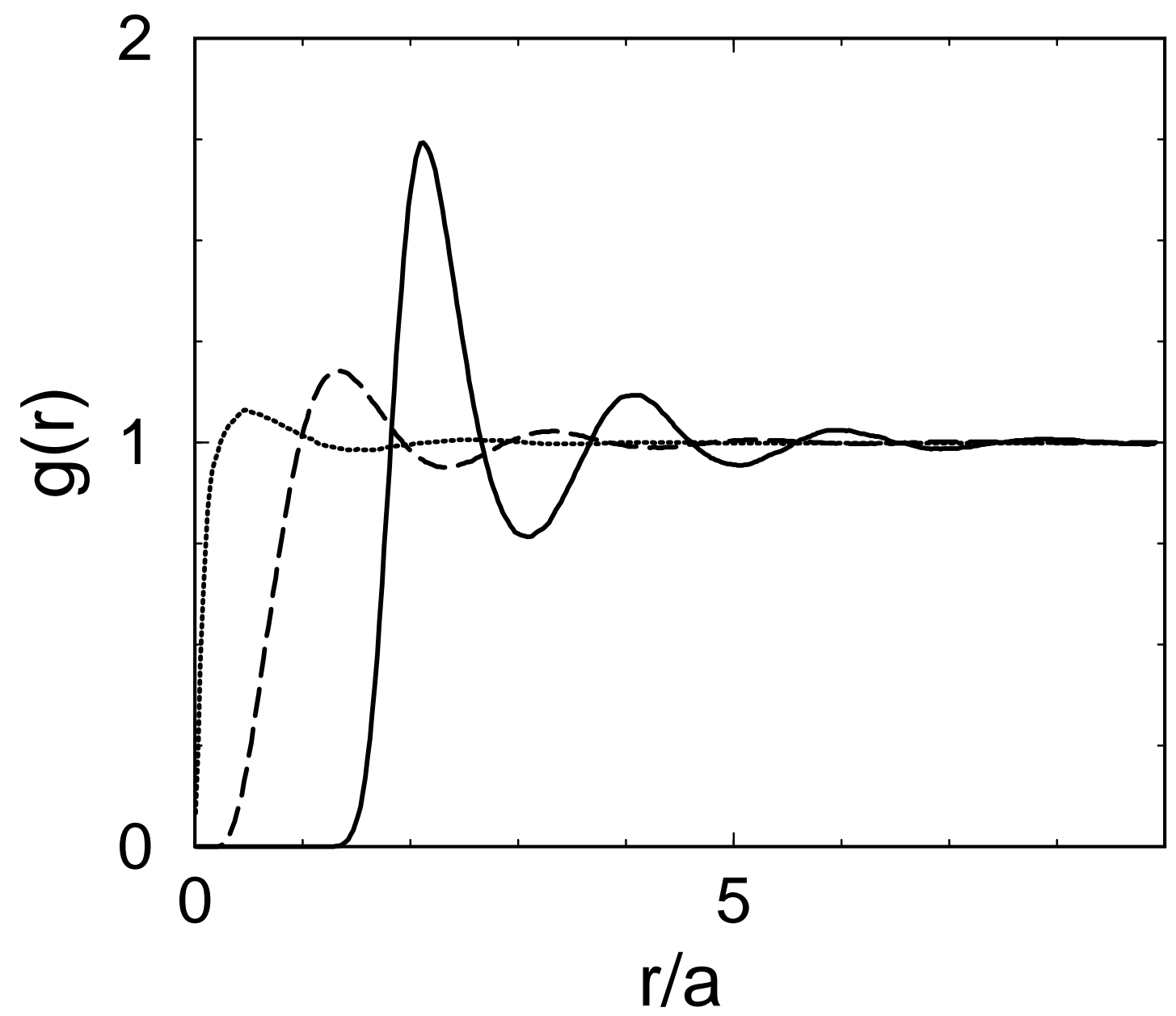

FIG. 2: Ion-ion pair correlation functions for $\Gamma=0.05, x_{A u}=0.5: D^{+}-D^{+}$(dotted line), $D^{+}-A u^{39+}($ dashed line), $A u^{39+}-A u^{39+}$ (solid line). 


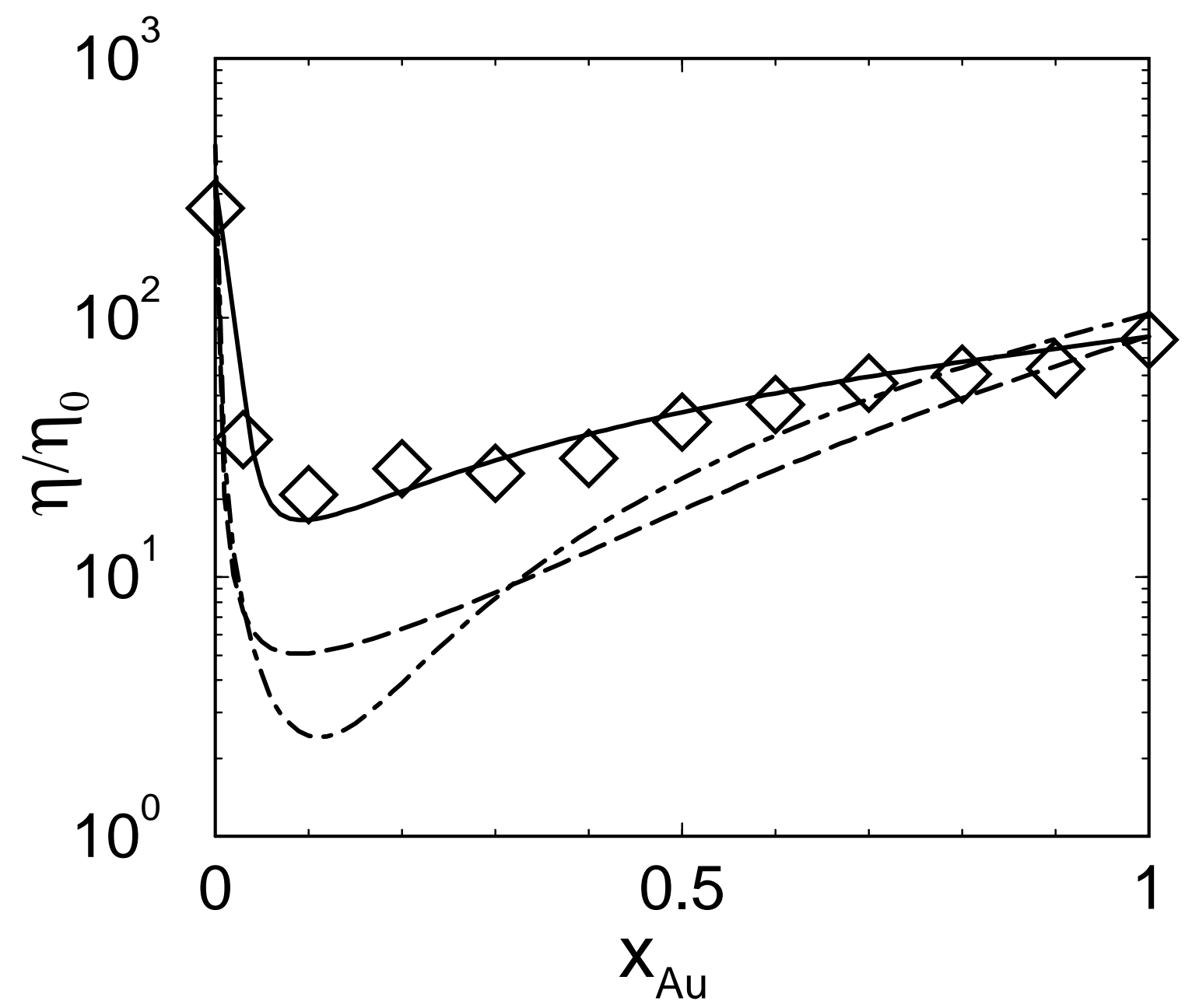

FIG. 3: Viscosity of the $D^{+}-A u^{39+}$ BIM at $\Gamma=0.05$ as a function of composition: simulations (diamonds), one-component model of Ref. [2] (dot-dashed line), one-component model using Eq. 11 for the OCP viscosity (dashed line), two-component model Eq. 14 (solid line). 


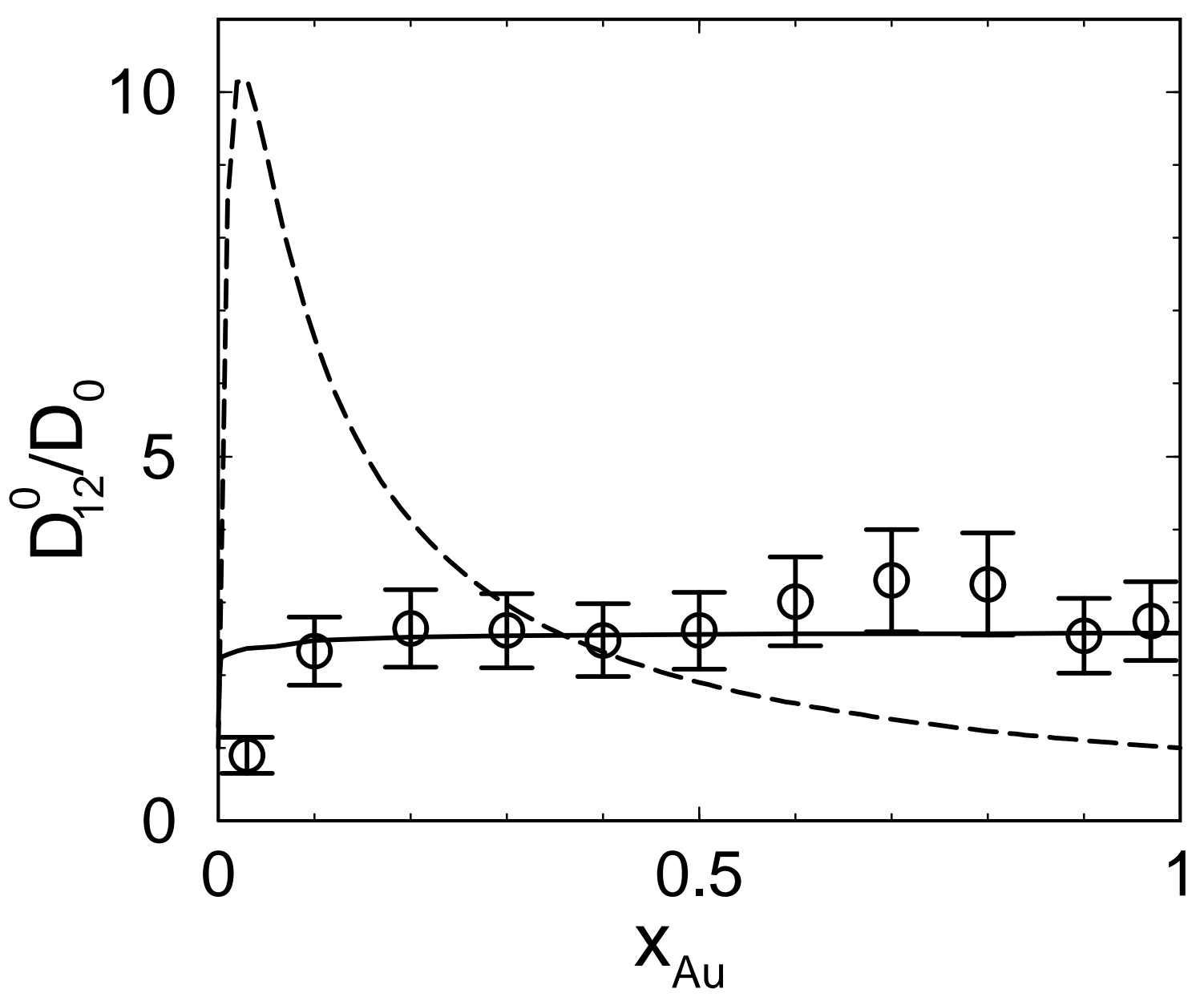

FIG. 4: Mutual diffusion constant contribution $D_{12}^{0}$ (see text) of the $D^{+}-A u^{39+}$ BIM at $\Gamma=0.05$ as a function of composition: simulations (circles), screened potential model of Ref. [32] (solid line). Thermodynamic prefactor $\left\langle Z^{2}\right\rangle /\langle Z\rangle^{2}($ dashed line). 